\title{
A practical approach based on stream of variation for positioning accuracy in precise linear motion stage

\author{
Hao Tang ${ }^{1, ~ a ~, ~ J i-a n ~ D u a n ~}{ }^{2, b}, Y u$ Zheng ${ }^{3, \mathrm{c}}$ and Baibing $\mathrm{Li}^{4, \mathrm{~d}}$ \\ ${ }_{1,2,3,4}$ State key laboratory of High-performance Complex Manufacture, \\ Central South University, Changsha, Hunan, 410083, China. \\ atanghaocsu@csu.edu.cn
}

Keywords: positioning accuracy; stream of variation; precise linear motion stage.

Abstract. This paper introduces a new analyzing approach on positioning accuracy (PA) in precise linear motion stage (PLMS). As one of the most important parameter, PA is affected by several reasons for the quality of PLMS, such as machining error, assembly error and controller accuracy etc. Conventional method only focuses on one aspect leading to positioning error (PE), which is not systematically and comprehensively. By adopting new analyzing approach, it is obviously understandable how errors from each part in PLMS give impact on PA and how PE accumulates. In this paper, the measuring result of PA is studied firstly. Then related factors affecting PA are concluded through analyzing characters in the measurement result of PA such as fluctuation, repeatability uncertainty and backlash. A series of analysis leading to these factors are introduced, including motor output, screw and assembly error, and an error model for PA in PLMS is established. A case study of one PLMS based on the new analysis procedure is developed, and corresponding experiments are carried out to collect the data. For one PLMS, this new approach on PA is not only beneficial for error compensation, but also helpful in design part. Furthermore, it is a systematic approach in PA analyzing procedure, which is also a new way to weigh PA in PLMS.

\begin{tabular}{l|l|l|l}
\hline \multicolumn{4}{c}{ Nomenclature } \\
\hline PLMS & Precise linear motion stage & PE & Positioning error \\
\hline PA $\left(P_{a}\right)$ & Positioning accuracy & $E_{w}$ & Measuring error \\
\hline LI & Laser interferometer & $D_{m}$ & Deviation of motor output \\
\hline$\eta$ & Motor efficiency & $S_{l}$ & Uncertainty of lead screw \\
\hline LCM & Laser Confocal Microscope & $\bar{q}$ & Mean deviation of lead \\
\hline$R_{m}$ & $\begin{array}{l}\text { Output of motor measuring } \\
\text { by pulse }\end{array}$ & $q_{i}$ & $\begin{array}{l}\text { Measured deviation of lead at } \\
\text { position } i\end{array}$ \\
\hline
\end{tabular}

\section{Introduction}

Precise linear motion stage (PLMS) plays an important role in engineering area, especially in high-accuracy motion system, i.e. optoelectronic packaging system ${ }^{[1-2]}$. Thus, for a PLMS, positioning accuracy (PA) is one of the most significant parameter. There are many reasons lead to positioning error (PE), including machining error, assembly error in installation, and other reasons, so PA is imperfect to meet the high-accuracy requirement. Conventional analysis only reveals which kind of factor will give impact PA on machine tools. However, the proportion of each factor is not in consideration. In this paper, a systematic analysis procedure about the factors on PA in PLMS is introduced.

Generally, machine tools and motion stage are typical precise system in engineering region, such as numerical center, coordinate measuring machine and precise stage. Brecher ${ }^{[3]}$ considered a complicated design for high-precision machine tools, but his work inclined to geometric error and Abby error, and positioning error was not emphasized. Landon ${ }^{[4]}$ analyzed positioning error of tools in milling, such as wokrpiece material, milling operation etc. However, some key points, i.e. slide and motor were not raised in content. The literatures mentioned above or concentrated on machine tools, 
or only studied one aspect of PA, which is hard to compensate PA completely. Thus, it is necessary to develop a systematic and comprehensive analysis on PA for PLMS.

Previous literatures have some drawbacks about PA in PLMS. First, the definition and derivation about PA only for machine tools in standards ${ }^{[5]}$, and these standards are developed for long range machines. Compared to machine tools, PLMS has some characters such as small size, low weight and short displacement, so thermal effect is not in consideration. It is necessary to establish a proper description for PLMS with short displacement. Second, some papers are focusing on PA in precise motion stage which is widely adopted in engineering industry. However, these scholars usually concentrated on controller accuracy and friction mechanism etc. Unlike calculating PE in final step, this method presents a systematic procedure about how much deviation of each part of PLMS takes.In a word, the analyzing procedure not only can help researchers compensate PA after installation, but also can benefit for engineers improving PA in design part based on analyzing results.

\section{Positioning Accuracy and methodology}

The typical structure of one PLMS is shown in Fig.1. It consists of motor, coupler, lead screw, guide-way, carriage and encoder etc. Some other stages may be different in one or two parts depending on special needs. Generally, PA is a key parameter as the character of motion system, and positioning error $(\mathrm{PE})$ is the results standing for PA in one measurement.

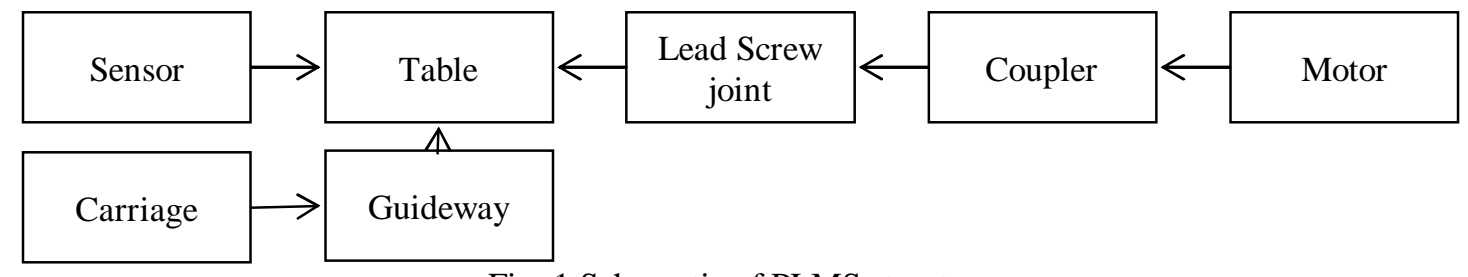

Fig. 1 Schematic of PLMS structure

Due to these imperfect reasons in manufacture and installation mentioned above, the accuracy of PLMS is affected. In order to understand how much percentage each error takes and how the PE accumulates, a systematic analysis is needed, which also can be used in other applications.

After introducing the analysis approach for PA propagation and accumulation, we need to know which factors and how these factors give impact on PA in PLMS. Therefore, a flow chart will be helpful to analyze PA systematically, as Fig.2 shows:

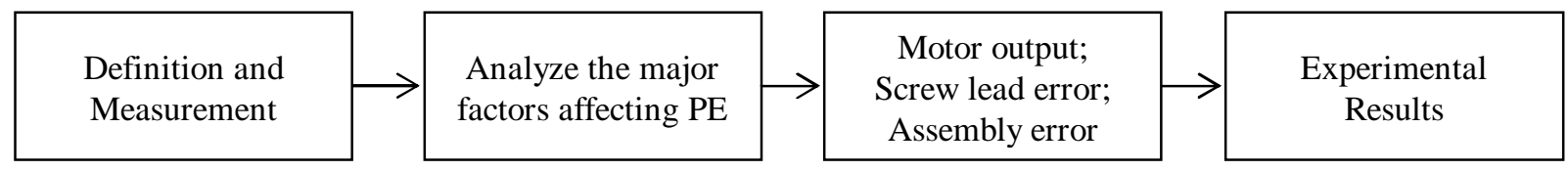

Fig. 2 Flow chart of analyzing procedure about PE

First, how to derive the PE based on standard should be clarified. This paper takes ISO 230-2 as reference standard, and uses ' 5 cycles test' to collect measurement data. It is helpful to understand the definition of PA by corresponding equations in ISO standard. Then, the factors resulting in PA need to be categorized into different groups. In order to analyze which factors are major reasons and how much percentage they takes, some analysis will be presented in following sections, and related experiments are raised to collect data.

Generally, a motion stage has 6 directional kinematic errors in movement. For a translational motion axis, the 6 kinematic error are named as positioning error, straightness error (vertical and horizontal), and angular error (pitch, yaw and roll) ${ }^{[5]}$. Different errors are resulted in different sources. Corresponding physical meaning for each error are also listed in Tab.1, respectively. 
Table 1 Physical meaning of kinematic errors in a translational axis

(Assuming along X-axis)

\begin{tabular}{|c|c|c|c|c|c|}
\hline Items & \multicolumn{5}{|c|}{ Error Components } \\
\hline & $\begin{array}{l}\text { Positioning } \\
\text { error }\end{array}$ & $\begin{array}{l}\text { Straightness } \\
\text { error }\end{array}$ & Pitch & Yaw & Roll \\
\hline $\begin{array}{l}\text { Physical } \\
\text { meaning }\end{array}$ & & & & & \\
\hline
\end{tabular}

Fig. 3 shows a typical distribution of collected data in standard measurement. It indicates that the parameter PA is determined by the measured value at each point. One thing should be pointed out that in the installation, the gap between different parts leads to backlash in bi-directional PE measurement. Thus, in studying PE, the backlash can represent the assembly error.

For PE, the accuracy of motor output stability and lead error of screw are important. Furthermore, the assembly error in installation not only gives impact on straightness error, but also on PA. It is necessary to adopt methods to measure the effects by motor output, lead screw and assembly error.

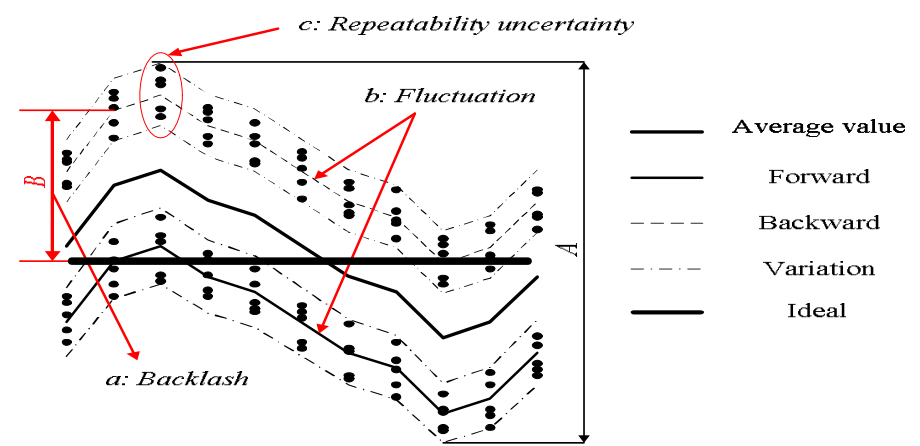

Fig. 3 Distribution of collected data in measurement (From ISO 230-2 standard)

Ideally, the measured result should be considered as a horizontal line (see in Fig.3), which means there is no backlash between forward and backward measurement, and the measure values are same for each measurement. However, we can infer that PA is affected by several reasons:

a. The difference between forward and backward measurement. Due to the gap between screw and nut in motion, some movements are regarded to compensate the gap instead of effective movement, which is represented by backlash.

b. The fluctuation of forward and backward measurements at each cycle. Theoretically, a smooth horizontal line is needed bi-directionally, which is standing for ideal situation without error. Due to unstable output at every step, there are deviations compared to ideal line. The deviations are determined by the lead error of screw, and some other minor factors.

c. The measured target position at every cycle, which is also named repeatability. It also contributes to PE by enlarging the error zone bi-directionally. Ideally, PLMS can achieve to the same position at every point. However, other factors except motor output can't result in this phenomenon. Thus, it is necessary to focus on the output of motor and assembly error between motor, couple and screw.

Besides, other factors also can lead to positioning error in motion process of PLMS, i.e. guide-way effect, controller accuracy and measurement accuracy etc.

Thus, the factors leading to PA categorized as Tab.2 shows: 
Table.2 Classification of factors affecting on PA

\begin{tabular}{|c|c|c|c}
\hline Error Category & $\begin{array}{c}\text { Difference between } \\
\text { forward and backward }\end{array}$ & $\begin{array}{c}\text { Fluctuation of each } \\
\text { cycle }\end{array}$ & $\begin{array}{c}\text { Repeatability at every } \\
\text { cycle }\end{array}$ \\
\hline Reason & Assembly error & Lead error & Accuracy of motor \\
\hline
\end{tabular}

\section{Case study}

\section{Structure of PLMS}

The structure of a typical PLMS is given as Fig.4 shows. (Suruga Seiki No.KS201-30RMS ). It consists of motor, guide-way, screw, table, and limit sensor mainly. In order to measure PA of the PLMS, laser interferometer (LI) is adopted in experiments.

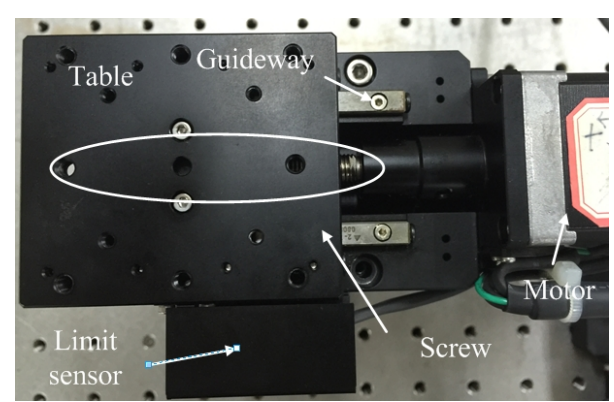

Fig.4 Structure of one studied PLMS

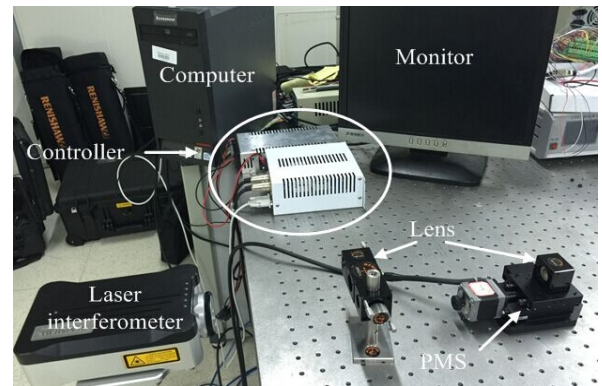

Fig.5 Layout of PA measuring experiment

Fig.5 shows layout of PA measuring experiment (including Laser interferometer, lens etc.). The humidity and temperature are controlled constantly.

By following ISO 230-2 measuring approach, the PA results are shown in Fig.6 and Tab.3.

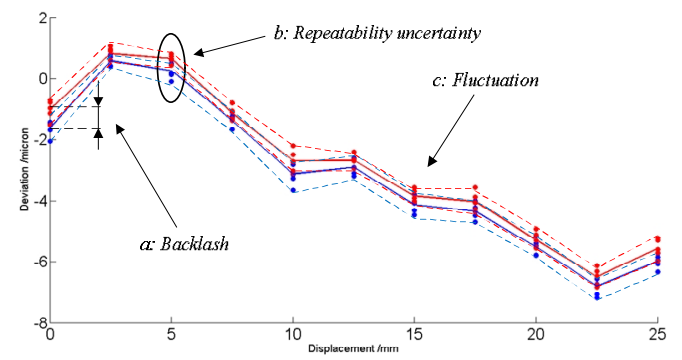

Fig. 6 Results of PE by following ISO 230-2 measurement

Table. 3 Results of PA measurement

\begin{tabular}{|l|c|c|c|c|}
\hline Items & Systematic deviation & Repeatability & Positioning Accuracy & Reversal value \\
\hline Value & $7.61(\mathrm{~m})$ & $1.49(\mathrm{~m})$ & $8.65(\mathrm{~m})$ & $0.60(\mathrm{~m})$ \\
\hline
\end{tabular}

In this measurement, the value of PA is $8.65 \mathrm{~m}$. Thermal effect can be ignored because the size of PLMS is small, the velocity is $0.5 \mathrm{~mm} / \mathrm{s}$, and temperature and humidity are constant. Backlash value is $0.60 \mathrm{~m}$, which is named as reversal value in ISO standard. This parameter can reflect assembly error between screw and nut due to the gaps in installation process. All equations and calculation process are based on ISO 230-2. Based on the results of PA, a series of analysis about PA are carried out in following content.

\section{Motor}

Motor, as driving part, can affect PA directly. The output stability of motor is very important to PA in PLMS. The motor in Fig.6 is a stepper motor. For one stage driven by stepper motor, step angle is a key parameter to measure the accuracy of motor output. Due to angle deviation in rotation, the accuracy of motor output is undermined. In order to measure the motor accuracy, some devices are adopted to measure the deviation of step angle in motion process. The existence of force ripple 
will result in accuracy degradation because motor output is not stable ${ }^{[6-7]}$. An experiment with oscilloscope is operated to measure the force ripple.

By measuring the variation of voltage, this sensor can calculate force ripple. Fig. 7 shows the curve in stable working state.

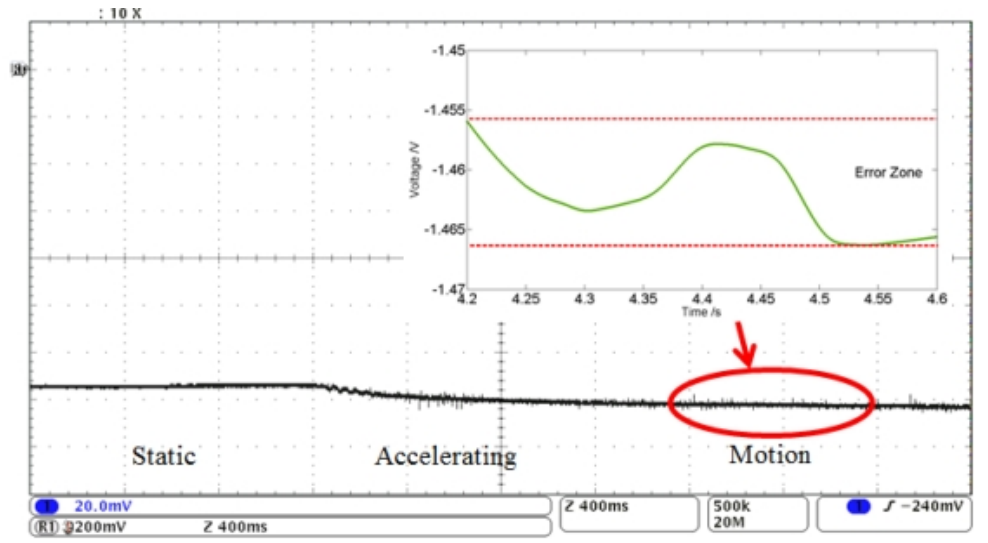

Fig. 7 Result for force ripple of motor output measured by oscilloscope and sensor

From the result in Fig.7, the force ripple is $0.011 \mathrm{~V}$, which means there is $0.37 \%$ fluctuation of force in motion process (Compare to the voltage at normal state). So, the force ripple can result in 1.85 m on PE. An equation can also denote the output deviation of motor:

$D_{m}=R_{m} \cdot \eta$

$R_{m}$ denotes the output of motor measuring by pulse, $\eta$ denotes the efficiency.

\section{Lead screw}

For a lead screw, the lead error, defined as actual mean travel deviation in ISO $3408-3^{[8]}$, is a significant parameter to weigh the quality of lead screw.

According to conventional method(laser interferometer is adopted), the screw measurement is same as PA measurement by using laser interferometer. It is difficult to extract lead error from PE. Thus, we adopt Laser Confocal Microscope (LCM) to collect the lead error, through measuring errors between two adjacent leads, as Fig.8 shows.

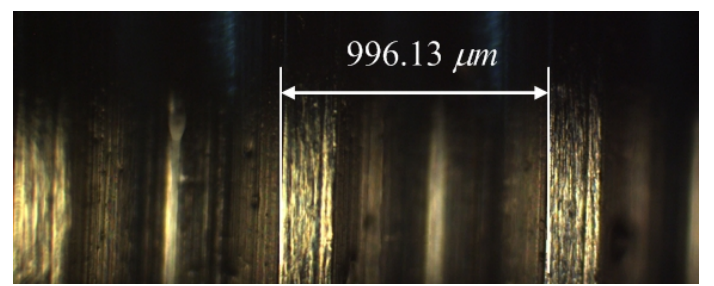

Fig. 8 Image of distance between two lead under LCM

Due to discrete data are collected, Eqn.4 can be adopted to reflect the character of screw:

$D_{l}=\sqrt{\frac{1}{n-1} \sum_{i=1}^{n}\left(q_{i}-\bar{q}\right)^{2}}$

where $D_{l}$ is lead error, $S_{l}$ is unidirectional uncertainty, $q_{i}$ is deviation of lead, $\bar{q}$ is mean deviation. 


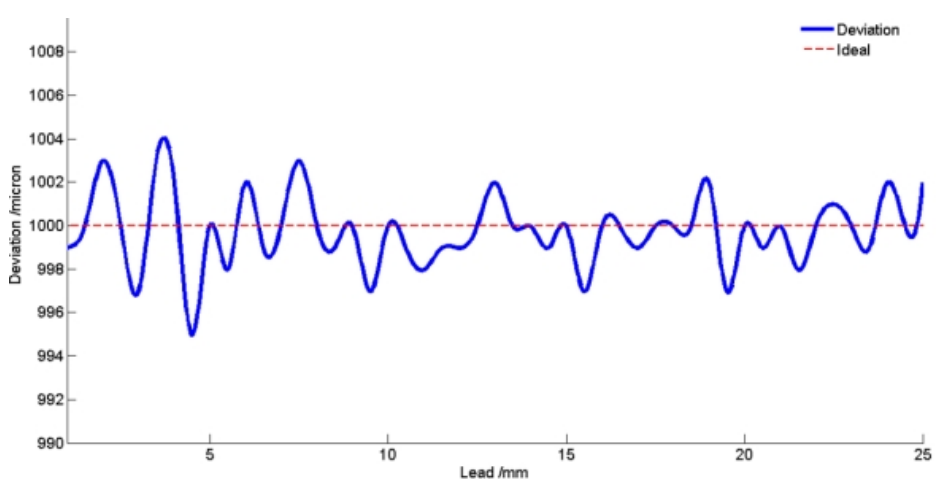

Fig. 9 Results of lead error in screw in useful path

Thus, the lead error is $3.23 \mu \mathrm{m}$ at $25 \mathrm{~mm}$ path based on Eq.2.

\section{Conclusion}

A systematic approach researching on PA in PLMS is developed in this paper, and a series of experiments are carried out to measure PA and the factors giving effect on PA in PLMS. Conventional method only analyzes one aspect on PA, and doesn't introduce a direct method and measurement about which error component affecting PA. Thus, it is necessary to understand which factors are primary and how much percentage these factors take. This new analysis approach has some following advantages:

1. It is obvious to understand the accuracy characteristics of PLMS, according to PA measurement result. There are 3 kinds of deviations affecting PA, including fluctuation, repeatability and backlash, which are related to lead error, motor output and assembly error.

2. The analyzing procedure not only can support error compensation with data, but also benefit in design part. The factors affecting PA and how PA accumulates are clearly.

A series of experiments about motor and screw are introduced. Compared to conventional method, these experiments can reflect the machining error and quality by using corresponding devices, such as sensor, laser interferometer and LCM. An error model for PA in PLMS is established. This error model includes 3 groups of error component, which can apply in other type of PLMS. By considering the structure difference, the parameters in error model can be substituted in cases.

\section{Acknowledgements}

This research is supported by the National Natural Science Foundation of China (Grant No.51075402 and No.50975293), the National High-Tech R\&D Program of China (Grant No.2012AA040406), and Postgraduate Innovation Program of Hunan province (CX2012B051).

\section{References}

[1] Lee KI, Lee JC, Yang SH.: Performance evaluation of five-DOF motion in ultra-precision linear stage, Int J Precis Eng \& Manuf. Vol. 15, No. 1, pp. 129-134, (2014).

[2] Li YM, Xiao SL,et al.: Design, modeling, control and experiment for a 2-DOF compliant micro-motion stage. Int J of Precis Eng \& Manuf. Vol. 15, Issue 4, pp. 735-744, (2014).

[3] Brecher C, Utsch P, Klar R, et al.: Compact design for high precision machine tools, Int J Mach tools \& Manuf. Vol. 50, pp. 328-334, (2015).

[4] Landon Y, Segonds S, Lascoumes P, et al.: Tool positioning error (TPE) characterization in milling, Int J Mach tools \& Manuf. Vol. 44, pp. 457-464, (2004).

[5] ISO 230-1,: Test Code for Machine Tools. Part 1. Geometric Accuracy of Machines Operating Under No-Load or Finishing Conditions, ISO, Geneva, (1996). 
[6] Zhao S, Tan K.: Adaptive feedforward compensation of force ripples in linear motor, Control Eng Pract. Vol. 13, pp. 1081-1092, (2005).

[7] Yamamoto A, Niino T, Higuchi T.: Modeling and identification of an electrostatic motor, Precis Eng. Vol. 30, pp. 104-113, (2006).

[8] ISO 3408-3: Ball screws. Part 3. Acceptance conditions and acceptance tests, ISO, Geneva, (2006). 